\title{
On boundary value problems for perturbed Hermitean matrix Dirac equations in a fractal domain
}

\author{
Ricardo Abreu Blaya Juan Bory Reyes
}

\begin{abstract}
We apply Hermitean circulant $(2 \times 2)$ matrix function theoretic results for direct account of the jump and Dirichlet type boundary value problems for solutions of perturbed Hermitean matrix Dirac equations in the case where the boundary of the considered domains is fractal.
\end{abstract}

\section{Introduction}

Euclidean Clifford analysis offers a function theory with the Dirac operator, which is an elegant generalization to higher dimensions of holomorphic functions in the complex plane. The best standard reference here is [6]. More recently Hermitean Clifford analysis emerged as a refinement of the Euclidean setting in the case of $\mathbb{R}^{2 n}$; it focuses on the concept of $h$-monogenic functions, i.e. null solutions of two complex Hermitean Dirac operators, which are invariant under the action of the unitary group. For a treatment of this function theory, we refer the reader for instance to $[7,8,9,22]$. Let us mention that holomorphic functions of several complex variables are a special case of Hermitian monogenic functions. In the recent papers $[1,2,3,4,10,11]$, the Hermitean Clifford analysis setting was further developed by following a circulant $(2 \times 2)$ matrix framework.

Received by the editors in October 2013 - In revised form in February 2014.

Communicated by H. De Schepper.

2010 Mathematics Subject Classification : 30G30, 30G35, 30E25.

Key words and phrases : complex Hermitean Clifford analysis; Helmholtz equation; boundary value problems. 
From both, mathematical convenience and physical relevancy, in the Euclidean Clifford algebras-based approach, there has been a great deal of activity in the study of a function theory associated to the perturbed Dirac operator, which components are annihilated by the Helmholtz operator. For basic results in this matter, we refer to $[5,14,21,20,24]$.

As explained in [1] it is possible to develop in a systematic way a matrix function theory associated to a Hermitean Helmholtz equation. The study of the jump and Dirichlet type boundary value problems in this Hermitean Helmholtz setting, but in fractal geometric context, is the main subject of this article.

\section{The Hermitean Clifford analysis setting}

Let $\left(e_{1}, \ldots, e_{m}\right)$ be an orthonormal basis of Euclidean space $\mathbb{R}^{m}$ and consider the complex Clifford algebra $\mathbb{C}_{m}$ constructed over $\mathbb{R}^{m}$. The non-commutative multiplication in $\mathbb{C}_{m}$ is governed by the rules:

$$
\begin{array}{rlrl}
e_{j}^{2} & =-1, \quad & j=1, \ldots, m \\
e_{j} e_{k}+e_{k} e_{j} & =0, \quad j \neq k
\end{array}
$$

$\mathbb{C}_{m}$ is then generated additively by elements of the form $e_{A}=e_{j_{1}} \ldots e_{j_{k}}$, where $A=\left\{j_{1}, \ldots, j_{k}\right\} \subset\{1, \ldots, m\}$ with $j_{1}<\cdots<j_{k}$, while for $A=\varnothing$, one puts $e_{\varnothing}=1$, the identity element. Any Clifford number $\lambda \in \mathbb{C}_{m}$ may thus be written as $\lambda=\sum_{A} \lambda_{A} e_{A}, \lambda_{A} \in \mathbb{C}$, its Hermitean conjugate $\lambda^{\dagger}$ being defined by $\lambda^{\dagger}=\sum_{A} \lambda_{A}^{c} \bar{e}_{A}$, where the bar denotes the real Clifford algebra conjugation, i.e. the main anti-involution for which $\bar{e}_{j}=-e_{j}$, and $\lambda_{A}^{c}$ stands for the complex conjugate of the complex number $\lambda_{A}$.

Euclidean space $\mathbb{R}^{m}$ is embedded in the Clifford algebra $\mathbb{C}_{m}$ by identifying $\left(x_{1}, \ldots, x_{m}\right)$ with the real Clifford vector $\underline{X}$ given by $\underline{X}=\sum_{j=1}^{m} e_{j} x_{j}$, for which $\underline{X}^{2}=-<\underline{X}, \underline{X}>=-|\underline{X}|^{2}$. The Fischer dual of $\underline{X}$ is the vector valued first order Dirac operator $\partial_{\underline{X}}=\sum_{j=1}^{m} e_{j} \partial_{x_{j}}$, factorizing the Laplacian: $\Delta_{m}=-\partial_{\underline{X}}^{2}$; it underlies the notion of monogenicity of a function, the higher dimensional counterpart of holomorphy in the complex plane.

Suppose $\Omega$ is a bounded domain, with sufficiently smooth boundary $\Gamma$, in $\mathbb{R}^{m}$. We will be interested in functions $g: \Omega \rightarrow \mathbb{C}_{m}$, which might be written as $g=\sum_{A} g_{A} e_{A}$ with $g_{A}$ complex valued. Property, such continuity, differentiability, integrability, and so on, are ascribed coordinate-wise. A Clifford algebra valued function $g$, defined and differentiable in $\Omega$ of $\mathbb{R}^{m}$, is then called (left) monogenic in $\Omega$ iff $\partial_{\underline{X}} g=0$ in $\Omega$.

The transition from Euclidean Clifford analysis as described above to the Hermitean Clifford setting is essentially based on the introduction of a complex structure $J$, i.e. an $\mathrm{SO}(m)$ element, satisfying $J^{2}=-\mathbf{1}_{m}$. Since such an element can not exist when the dimension of the vector space is odd, we put $m=2 n$ from now on. In terms of the chosen orthonormal basis, a possible realization of the complex structure is $J\left[e_{2 j-1}\right]=-e_{2 j}$ and $J\left[e_{2 j}\right]=e_{2 j-1}, j=1, \ldots, n$. Two projection operators $\pm \frac{1}{2}\left(\mathbf{1}_{2 n} \pm i J\right)$ associated to $J$ then produce the main objects of Hermitean 
Clifford analysis by acting upon the corresponding objects in the Euclidean setting.

The vector space $\mathbb{C}^{2 n}$ thus decomposes as $W^{+} \oplus W^{-}$into two isotropic subspaces. The real Clifford vector is now denoted

$$
\underline{X}=\sum_{j=1}^{n}\left(e_{2 j-1} x_{2 j-1}+e_{2 j} x_{2 j}\right)
$$

with the corresponding Dirac operator

$$
\partial_{\underline{X}}=\sum_{j=1}^{n}\left(e_{2 j-1} \partial_{x_{2 j-1}}+e_{2 j} \partial_{x_{2 j}}\right)
$$

while we will also consider their so-called 'twisted' counterparts, obtained through the action of $J$, i.e.

$$
\begin{aligned}
\underline{X} & =\sum_{j=1}^{n}\left(e_{2 j-1} x_{2 j}-e_{2 j} x_{2 j-1}\right) \\
\partial_{\underline{X}} & =\sum_{j=1}^{n}\left(e_{2 j-1} \partial_{x_{2 j}}-e_{2 j} \partial_{x_{2 j-1}}\right)
\end{aligned}
$$

As was the case with $\partial_{\underline{X}}$, a notion of monogenicity may be associated in a natural way to $\partial_{\underline{X}}$ as well. The projections of the vector variable $\underline{X}$ on the spaces $W^{ \pm}$ then yield the Hermitean Clifford variables $\underline{Z}$ and $\underline{Z}^{\dagger}$, given by

$$
\underline{Z}=\frac{1}{2}(\underline{X}+i \underline{X} \mid) \text { and } \underline{Z}^{\dagger}=-\frac{1}{2}(\underline{X}-i \underline{X} \mid)
$$

and those of the Dirac operator $\partial_{\underline{X}}$ yield (up to a factor) the Hermitean Dirac operators $\partial_{\underline{Z}}$ and $\partial_{\underline{Z}^{\dagger}}$, given by

$$
\partial_{\underline{Z}}=-\frac{1}{4}\left(\partial_{\underline{X}}-i \partial_{\underline{X}}\right) \quad \text { and } \quad \partial_{\underline{Z}^{+}}=\frac{1}{4}\left(\partial_{\underline{X}}+i \partial_{\underline{X} \mid}\right)
$$

The Hermitean vector variables and Dirac operators are isotropic, i.e. $(\underline{Z})^{2}=\left(\underline{Z}^{\dagger}\right)^{2}=0$ and $\left(\partial_{\underline{Z}}\right)^{2}=\left(\partial_{\underline{Z}^{+}}\right)^{2}=0$, whence the Laplacian allows for the decomposition $\Delta_{2 n}=4\left(\partial_{\underline{Z}} \partial_{\underline{Z}^{+}}+\partial_{\underline{Z^{+}}} \partial_{\underline{Z}}\right)$. These objects lie at the core of the Hermitean function theory by means of the following definition (see e.g. $[7,9]$ ).

Definition 1. A continuously differentiable function $g$ in $\Omega \subset \mathbb{R}^{2 n}$ with values in $\mathbb{C}_{2 n}$ is called left Hermitean monogenic (or left h-monogenic) in $\Omega$, iff it satisfies the system $\partial_{\underline{Z}} g=0=\partial_{\underline{Z}^{+}} g$ or the equivalent system $\partial_{\underline{X}} g=0=\partial_{\underline{X}} \mid g$.

In this definition lies the origin of the statement that h-monogenic functions constitute a refinement of monogenic ones. In a similar way right h-monogenicity is defined. Functions which are both left and right h-monogenic are called twosided h-monogenic. 


\section{Function theory for perturbed Hermitean Dirac operators. A matrix approach.}

For the convenience of the reader we repeat the relevant material from [1] without proofs, thus making our exposition self-contained.

For $a \in \mathbb{R}$, a fundamental solution for the Helmholtz operator $\Delta_{2 n}+a^{2}$ in $\mathbb{R}^{2 n}$ is given by the formula

$$
\theta_{a}(\underline{X}):=-\frac{1}{(4 \pi)^{n}} \int_{0}^{\infty} \exp \left(a^{2} t-\frac{|\underline{X}|^{2}}{4 t}\right) \frac{d t}{t^{n}}, a \neq 0,
$$

whose main properties are collected in [21, Proposition 3.1].

Then fundamental solutions to the perturbed Euclidean Dirac operators $\partial_{\underline{X}}+a$ and $\partial_{\underline{X}}+a$ are respectively

$$
\begin{aligned}
E_{a}(\underline{X}) & =-\left[\left(\partial_{\underline{X}}-a\right) \theta_{a}(\underline{X})\right], \\
\left.E\right|_{a}(\underline{X}) & =-\left[\left(\partial_{\underline{X}}-a\right) \theta_{a}(\underline{X})\right] .
\end{aligned}
$$

The Hermitian counterparts of fundamental solutions $\left(E_{a},\left.E\right|_{a}\right)$ are give by

$$
\begin{gathered}
\mathcal{E}_{A}=-\left(E_{a}+\left.i E\right|_{a}\right), \\
\mathcal{E}_{A}^{\dagger}=\left(E_{a}-\left.i E\right|_{a}\right),
\end{gathered}
$$

or, more explicitly,

$$
\begin{gathered}
\mathcal{E}_{A}(\underline{Z})=4\left[\left(\partial_{\underline{Z}^{\dagger}}+A^{\dagger}\right) \theta_{a}(\underline{Z})\right], \\
\mathcal{E}_{A}^{\dagger}(\underline{Z})=4\left[\left(\partial_{\underline{Z}}-A\right) \theta_{a}\left(\underline{Z}^{\dagger}\right)\right],
\end{gathered}
$$

where

$$
A=-\frac{1}{4}(a-i a), \quad A^{\dagger}=\frac{1}{4}(a+i a) .
$$

The Hermitean Cauchy kernels $\mathcal{E}_{A}$ and $\mathcal{E}_{A}^{\dagger}$ are not the fundamental solutions to the respective perturbed Hermitean Dirac operators $\partial_{\underline{Z}}$ and $\partial_{\underline{Z}^{+}}$. However, the circulant $(2 \times 2)$ matrix

$$
\mathcal{E}_{A}:=\left(\begin{array}{cc}
\mathcal{E}_{A} & \mathcal{E}_{A}^{+} \\
\mathcal{E}_{A}^{+} & \mathcal{E}_{A}
\end{array}\right)
$$

can be thought of as a fundamental solution of the operator

$$
\mathcal{D}_{\left(\underline{Z}, \underline{Z}^{\dagger}\right)}^{\mathcal{A}}=\mathcal{D}_{\left(\underline{Z}, \underline{Z}^{\dagger}\right)}+\mathcal{A}
$$

where

$$
\mathcal{D}_{\left(\underline{Z}, \underline{Z}^{\dagger}\right)}=\left(\begin{array}{ll}
\partial_{\underline{Z}} & \partial_{\underline{Z}^{\dagger}} \\
\partial_{\underline{Z}^{\dagger}} & \partial_{\underline{Z}}
\end{array}\right), \mathcal{A}=\left(\begin{array}{cc}
A & A^{\dagger} \\
A^{\dagger} & A
\end{array}\right),
$$

i.e. $\mathcal{D}_{\left(\underline{Z}, \underline{Z}^{\dagger}\right)}^{\mathcal{A}} \mathcal{E}_{A}=\delta$, with $\delta=\left(\begin{array}{ll}\delta & 0 \\ 0 & \delta\end{array}\right)$ and $\delta$ being the Dirac delta distribution.

This fact motivates to introduce the following notion. 
Definition 2. Let $g_{1}, g_{2}$ be continuously differentiable functions defined in $\Omega \subset \mathbb{R}^{2 n}$ and taking values in $\mathbb{C}_{2 n}$, and consider the matrix function

$$
\boldsymbol{G}_{2}^{1}=\left(\begin{array}{ll}
g_{1} & g_{2} \\
g_{2} & g_{1}
\end{array}\right)
$$

Then $\boldsymbol{G}_{2}^{1}$ is called (left) $\mathbf{H}^{\mathcal{A}}$-monogenic in $\Omega$ if and only if it satisfies in $\Omega$ the system $\mathcal{D}_{\left(\underline{Z}, \underline{Z}^{\dagger}\right)}^{\mathcal{A}} G_{2}^{1}=\boldsymbol{O}$. Here $\boldsymbol{O}$ denotes the matrix with zero entries.

Remark 3.1. The important case of $\mathbf{H}$-monogenic functions (see $[1,2,3,4,10,11]$ ) arises when we take $\mathcal{A}=\boldsymbol{O}$ in the above definition.

Notions of continuity, differentiability and integrability of a circulant matrix function $G_{2}^{1}$ are introduced through the corresponding notions for its entries. In particular, we will need to define in this way the classes $\mathcal{C}^{0, v}(\mathbf{E})$ and $\mathbf{L}_{p}(\mathbf{E})$ of, respectively, Hölder continuous and $p$-integrable circulant matrix functions over some suitable subset $\mathbf{E}$ of $\mathbb{R}^{2 n}$. However, introducing the non-negative function

$$
\left\|G_{2}^{1}(\underline{X})\right\|=\max \left\{\left|g_{1}(\underline{X})\right|,\left|g_{2}(\underline{X})\right|\right\}
$$

these classes of circulant matrix functions may be defined by means of the traditional conditions

$$
\sup _{\underline{X}, \underline{Y} \in \mathbf{E} ; \underline{X} \neq \underline{Y}} \frac{\left\|G_{2}^{1}(\underline{X})-G_{2}^{1}(\underline{Y})\right\|}{|\underline{X}-\underline{Y}|^{v}}<+\infty .
$$

and

$$
\int_{\mathbf{E}}\left\|G_{2}^{1}(\underline{X})\right\|^{p}<+\infty
$$

In the sequel we will also use the notations $\mathbf{C}^{1}(\mathbf{E})$ for circulant matrix functions whose entries are, together with their derivatives, continuous in $\mathbf{E}$ and the Sobolev space $\mathbf{W}_{p}^{1}(\mathbf{E})$ of $\mathbf{L}_{p}(\mathbf{E})$-matrix whose entries are weakly differentiable in the sense of Sobolev.

The fundamental solution of the operator $\mathcal{D}_{\left(\underline{Z}, \underline{z}^{\dagger}\right)}^{\mathcal{A}}$ now allows us to explicitly express and to introduce the corresponding Teodorescu operator:

$$
\mathcal{T}_{\Omega}^{\mathcal{A}} G_{2}^{1}(\underline{Y}):=-\int_{\Omega} \mathcal{E}_{A}(\underline{Z}-\underline{V}) G_{2}^{1}(\underline{X}) d W\left(\underline{Z}, \underline{Z}^{\dagger}\right),
$$

where $\underline{V}=\frac{1}{2}(\underline{Y}+i \underline{Y} \mid)$ and $d W\left(\underline{Z}, \underline{Z}^{\dagger}\right)$ is -up to a constant complex factor- the volume element given by

$$
d V \underline{X}=(-1)^{\frac{n(n-1)}{2}}\left(\frac{i}{2}\right)^{n} d W\left(\underline{Z}, \underline{Z}^{\dagger}\right)
$$

For further use, let us mention some mapping properties of the operator $\mathcal{T}_{\boldsymbol{\Omega}}^{\mathcal{A}}$, as formulated in the following theorem. The proof is an adaptation of results proved in $[5,15,16]$ to our matrix context. 
Theorem 1. Let $\Omega \subset \mathbb{R}^{2 n}$ and $p>2 n$. Then

- $\mathcal{T}_{\boldsymbol{\Omega}}^{\mathcal{A}}: \mathbf{L}_{p}(\Omega) \longrightarrow \mathbf{W}_{p}^{1}(\Omega)$ is continuous.

- If $\mathbf{G}_{2}^{1} \in \mathbf{L}_{p}(\Omega)$, then

$$
\mathcal{D}_{\left(\underline{Z}, \underline{Z}^{+}\right)}^{\mathcal{A}} \mathcal{T}_{\Omega}^{\mathcal{A}} G_{2}^{1}(\underline{Y})= \begin{cases}(-1)^{\frac{n(n+1)}{2}}(2 i)^{n} G_{2}^{1}(\underline{Y}), & \underline{Y} \in \Omega, \\ \boldsymbol{O}, & \underline{Y} \in \mathbb{R}^{2 n} \backslash \bar{\Omega} .\end{cases}
$$

- $\mathcal{T}_{\mathbf{\Omega}}^{\mathcal{A}}: \mathbf{L}_{p}(\Omega) \longrightarrow \mathbf{C}^{0, v}\left(\mathbb{R}^{2 n}\right)$ is continuous for any $0<v \leq \frac{p-2 n}{p}$.

For our purposes, let us reformulate in matrix form a very important theorem from real analysis: the Whitney extension theorem [23].

Theorem 2 (Whitney Extension Theorem). Let $\mathbf{E} \subset \mathbb{R}^{2 n}$ be compact and $\boldsymbol{G}_{2}^{1} \in \mathcal{C}^{0, v}(\mathbf{E})$. Then, there exists a compactly supported matrix function $\widetilde{\mathbf{G}}_{2}^{1}$ satisfying

(i) $\left.\widetilde{G}_{2}^{1}\right|_{\mathbf{E}}=G_{2}^{1}$;

(ii) $\widetilde{G}_{2}^{1} \in \mathbf{C}^{1}\left(\mathbb{R}^{2 n} \backslash \mathbf{E}\right)$;

(iii) $\left\|\mathcal{D}_{\left(\underline{Z}, \underline{Z}^{\dagger}\right)} \widetilde{G}_{2}^{1}(\underline{X})\right\| \leq c \operatorname{dist}(\underline{X}, \mathbf{E})^{v-1}$, for $\underline{X} \in \mathbb{R}^{2 n} \backslash \mathbf{E}$.

\section{Box dimension and $d$-summable sets in $\mathbb{R}^{2 n}$}

We now briefly recall some specific preparatory material concerning fractal sets in $\mathbb{R}^{2 n}$.

Let $\mathbf{E}$ be an arbitrary subset of $\mathbb{R}^{2 n}$. Then for any $s \geq 0$ its Hausdorff measure $\mathcal{H}^{s}(\mathbf{E})$ may be defined by

$$
\mathcal{H}^{s}(\mathbf{E})=\lim _{\delta \rightarrow 0} \inf \left\{\sum_{k=1}^{\infty}\left(\operatorname{diam} B_{k}\right)^{s}: \mathbf{E} \subset \bigcup_{k=1}^{\infty} B_{k}, \operatorname{diam} B_{k}<\delta\right\}
$$

the infimum being taken over all countable $\delta$-coverings $\left\{B_{k}\right\}$ of $\mathbf{E}$ with open or closed balls. For $s=2 n$, the Hausdorff measure $\mathcal{H}^{2 n}$ coincides, up to a positive multiplicative constant, with the Lebesgue measure $\mathcal{L}^{2 n}$ in $\mathbb{R}^{2 n}$.

Now, let $\mathbf{E}$ be compact. The Hausdorff dimension $\alpha_{H}(\mathbf{E})$ of $\mathbf{E}$ is then defined as the infimum of all $s \geq 0$ such that $\mathcal{H}^{s}(\mathbf{E})<+\infty$. For more details concerning the Hausdorff measure and dimension we refer to [12]. Frequently however, see [19], the so-called box dimension is used, defined for a compact set $\mathbf{E} \subset \mathbb{R}^{2 n}$ as

$$
\alpha(\mathbf{E})=\lim _{\varepsilon \rightarrow 0} \sup \frac{\log N_{\mathbf{E}}(\varepsilon)}{-\log \varepsilon}
$$

where $\mathbf{N}_{\mathbf{E}}(\varepsilon)$ stands for the minimal number of $\varepsilon$-balls needed to cover $\mathbf{E}$. Note that the limit above remains unchanged if $\mathbf{N}_{\mathbf{E}}(\varepsilon)$ is replaced by the number of $k$-cubes, with $2^{-k} \leq \varepsilon<2^{-k+1}$, intersecting $\mathbf{E}$. A cube $Q$ is called a $k$-cube if it is 
of the form $\left[l_{1} 2^{-k},\left(l_{1}+1\right) 2^{-k}\right] \times \cdots \times\left[l_{2 n} 2^{-k},\left(l_{2 n}+1\right) 2^{-k}\right]$, where $k$ and $l_{1}, \ldots, l_{2 n}$ are integers. The box dimension and the Hausdorff dimension of a given compact set $\mathbf{E}$ can be equal, which is for instance the case for the so-called $(2 n-1)$ rectifiable sets (see [13]), but in general we have that $\alpha_{H}(\mathbf{E}) \leq \alpha(\mathbf{E})$.

The following geometric notion was introduced in [17], and is essential in their method of integrating a form over a fractal boundary.

Definition 3. The compact set $\mathbf{E}$ is said to be d-summable if the improper integral $\int_{0}^{1} N_{\mathbf{E}}(x) x^{d-1} d x$ converges.

Lemma 1 ([17]). It holds that

(i) any $d$-summable set $\mathbf{E}$ has box dimension $\alpha(\mathbf{E}) \leq d$;

(ii) if $\alpha(\mathbf{E})<d$, then $\mathbf{E}$ is d-summable;

(iii) if $\mathbf{E}$ is $d$-summable, then it is also $(d+\varepsilon)$-summable for every $\varepsilon>0$.

In what follows, we will take $\Omega \subset \mathbb{R}^{2 n}$ to be a Jordan domain, i.e. a bounded oriented connected open subset of $\mathbb{R}^{2 n}$, the boundary $\Gamma$ of which is a compact topological surface. For our purpose, we will assume that the Hausdorff and box dimensions of $\Gamma$ satisfy $2 n-1 \leq \alpha_{H}(\Gamma) \leq \alpha(\Gamma)<2 n$. Note that this includes the case when $\Gamma$ is fractal in the sense of Mandelbrot, i.e. when $2 n-1<\alpha_{H}(\Gamma)$. Under these conditions, there will always exist $d \in[2 n-1,2 n[$ such that $\Gamma$ is $d$-summable, see Lemma 1 .

We will also need the so-called Whitney decomposition of $\Omega$, which we will only recall briefly; for details we refer to [23]. Consider the lattice $\mathbb{Z}^{2 n}$ in $\mathbb{R}^{2 n}$ and the collection of closed unit cubes defined by it; let $\mathcal{M}_{1}$ be the mesh consisting of those unit cubes having a non-empty intersection with $\Omega$. We then recursively define the meshes $\mathcal{M}_{k}, k=2,3, \ldots$, each time bisecting the sides of the cubes of the previous one. The cubes in $\mathcal{M}_{k}$ thus have side length $2^{-k+1}$ and diameter $|Q|=\sqrt{2 n} 2^{-k+1}$. We then define, for $k=2,3, \ldots$,

$$
\begin{array}{r}
\mathcal{W}^{1}=\left\{Q \in \mathcal{M}_{1} \mid \text { all neighbour cubes of } Q \text { belong to } \Omega\right\} \\
\mathcal{W}^{k}=\left\{Q \in \mathcal{M}_{k} \mid \text { all neighbour cubes of } Q \text { belong to } \Omega\right. \text {, and } \\
\left.\qquad \nexists Q^{*} \in \mathcal{W}^{k-1}: Q \subset Q^{*}\right\}
\end{array}
$$

for which it can be proven (see [23]) that

$$
\Omega=\bigcup_{k=1}^{+\infty} \mathcal{W}^{k}=\bigcup_{k=1}^{+\infty} \bigcup_{Q \in \mathcal{W}^{k}} Q \equiv \bigcup_{Q \in \mathcal{W}} Q,
$$

all cubes $Q$ in the Whitney decomposition $\mathcal{W}$ of $\Omega$ having disjoint interiors.

We then have the following relation between the $d$-summability of the boundary $\Gamma$ and the Whitney decomposition of $\Omega$.

Lemma 2 ([17]). If $\Omega$ is a Jordan domain of $\mathbb{R}^{2 n}$ and its boundary $\Gamma$ is d-summable, then the expression $\sum_{Q \in \mathcal{W}}|Q|^{d}$, called the d-sum of the Whitney decomposition $\mathcal{W}$ of $\Omega$, is finite. 


\section{Boundary value problems}

The theory of perturbed Dirac operator has been proven to be a very efficient toolkit to treat large classes of boundary value problems in almost all areas of physics and engineering such as for instance, electromagnetism, optic, elasticity, fluid dynamics, hydroacustic and geophysic, see [5, 14, 15, 18, 20, 21, 24] and many other books and articles.

It is worth pointing out that the study of boundary value problems for such physical models has been confined to domains with sufficiently smooth boundary, Lipschitz domains in the worst case scenario, see for instance [20, 21]. Of course, for the purpose of numerous applications, and for sake of pure mathematical generality, it would be of great interest to be able to lift these geometric restrictions. In this section we deal with two important boundary value problems in the theory of perturbed Hermitean matrix Dirac equation in the more challenging case of domains with fractal boundaries.

In what follows, we will take $\Omega \subset \mathbb{R}^{2 n}$ to be a Jordan domain with $d$-summable boundary $\Gamma, d \in[2 n-1,2 n[$. For brevity we adopt the temporary notation $\Omega_{+}=\Omega, \Omega_{-}=\mathbb{R}^{2 n} \backslash\{\Omega \cup \Gamma\}$.

We start by considering the jump problem for $\mathbf{H}^{\mathcal{A}}$-monogenic functions on fractal surfaces in $\mathbb{R}^{2 n}$, that is, the problem of reconstructing a $\mathbf{H}^{\mathcal{A}}$-monogenic function $\boldsymbol{\Xi}_{2}^{\mathbf{1}}$ in $\mathbb{R}^{2 n} \backslash \Gamma$ vanishing at infinity and having a prescribed jump $G_{2}^{1}$ across $\Gamma$, i.e.

$$
\boldsymbol{\Xi}_{\mathbf{2}}^{\mathbf{1}^{+}}-\boldsymbol{\Xi}_{\mathbf{2}}^{\mathbf{1}^{-}}=G_{2}^{1} \text {, in } \Gamma, \boldsymbol{\Xi}_{\mathbf{2}}^{\mathbf{1}}(\infty)=O,
$$

where $\boldsymbol{\Xi}_{\mathbf{2}}^{\mathbf{1}^{ \pm}}(\underline{X})=\lim _{\Omega_{ \pm} \ni \underline{Y} \rightarrow \underline{X}} \boldsymbol{\Xi}_{\mathbf{2}}^{\mathbf{1}}(\underline{Y})$.

The next step will be to consider the Dirichlet type problem

$$
\begin{gathered}
\mathcal{D}_{\left(\underline{Z}, \underline{Z}^{\dagger}\right)}^{\mathcal{A}} \boldsymbol{\Xi}_{2}^{\mathbf{1}}=F_{2}^{1}, \quad \text { in } \Omega, \\
\boldsymbol{\Xi}_{\mathbf{2}}^{\mathbf{1}}=G_{2}^{1}, \quad \text { on } \Gamma .
\end{gathered}
$$

The critical technical results to investigate the solvability of these boundary value problems are the following

Proposition 1. Let $\Omega \subset \mathbb{R}^{2 n}$ with d-summable boundary $\Gamma$ such that $d \in[2 n-1,2 n[$, and let $\boldsymbol{G}_{2}^{1} \in \mathcal{C}^{0, v}(\Gamma)$, then $\mathcal{D}_{\left(\underline{Z}, \underline{Z}^{\dagger}\right)}^{\mathcal{A}} \widetilde{G}_{2}^{1} \in \mathbf{L}_{p}(\Omega)$ for any $0<p<\frac{2 n-d}{1-v}$.

Proof.

By definition, $\mathcal{D}_{\left(\underline{Z}, \underline{Z}^{\dagger}\right)}^{\mathcal{A}} \widetilde{G}_{2}^{1}=\mathcal{D}_{\left(\underline{Z}, \underline{Z}^{\dagger}\right)} \widetilde{G}_{2}^{1}+\mathcal{A} \widetilde{G}_{2}^{1}$. Since $\widetilde{G}_{2}^{1} \in \mathcal{C}^{0, v}(\bar{\Omega})$ it follows that

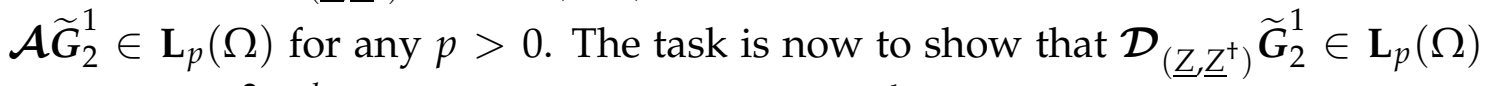
for any $p<\frac{2 n-d}{1-v}$. To this end, let $\mathcal{W}=\bigcup_{k=1}^{\infty} \mathcal{W}^{k}$ be the Whitney decomposition of $\Omega$. Then we have

$$
\begin{aligned}
\int_{\Omega}\left\|\mathcal{D}_{\left(\underline{Z}, \underline{Z}^{\dagger}\right)} \widetilde{\boldsymbol{G}}_{2}^{1}(\underline{Y})\right\|^{p} d V(\underline{Y}) & =\sum_{Q \in \mathcal{W}} \int_{\Omega}\left\|\mathcal{D}_{\left(\underline{Z}, \underline{Z}^{\dagger}\right)} \widetilde{\boldsymbol{G}}_{2}^{1}(\underline{Y})\right\|^{p} d V(\underline{Y}) \\
& \leq c \sum_{Q \in \mathcal{W}} \int_{Q} \operatorname{dist}(\underline{Y}, \Gamma)^{-p(1-v)} d V(\underline{Y})
\end{aligned}
$$


the last inequality following from Theorem 2 (iii). By construction of the Whitney decomposition of $\Omega$, we have that, for any $Q \in \mathcal{W}$,

$$
\operatorname{dist}(\underline{Y}, \Gamma) \geq \frac{|Q|}{\sqrt{2 n}}, \quad \forall \underline{Y} \in Q
$$

see also [23], whence

$$
\int_{\Omega}\left\|\mathcal{D}_{\left(\underline{Z}, \underline{Z}^{\dagger}\right)} \widetilde{\boldsymbol{G}}_{2}^{1}(\underline{Y})\right\|^{p} \leq c \sum_{Q \in \mathcal{W}}|Q|^{2 n-p(1-v)} .
$$

The finiteness of the last sum follows, on account of Lemma 2 , from the $d$-summability of $\Gamma$, together with the fact that $2 n-p(1-v)>d$.

Proposition 2. Let be $\Omega \subset \mathbb{R}^{2 n}$ with $d$-summable boundary $\Gamma$ such that $d \in\left[2 n-1,2 n\left[\right.\right.$. Furthermore, let $\frac{d}{2 n}<v \leq 1$ and consider $G_{2}^{1} \in \mathcal{C}^{0, v}(\Gamma)$. Then $\mathcal{T}_{\Omega}^{\mathcal{A}} \mathcal{D}_{\left(\underline{Z} \underline{Z}^{\dagger}\right)}^{\mathcal{A}} \widetilde{G}_{2}^{1}$ belongs to $\mathcal{C}^{0, \mu}(\Gamma)$, whenever

$$
\mu<\frac{2 n v-d}{2 n-d}
$$

Proof.

Since $v>\frac{d}{2 n}$ implies that $2 n<\frac{2 n-d}{1-v}$, we may choose $p$ such that $2 n<p<\frac{2 n-d}{1-v}$. The proof being a consequence of Theorem 1 and Proposition 1.

\subsection{Treatment of the problem (2)}

The following theorem provides a criterion for the solvability of the boundary value problem (2). Compare with the case $\mathcal{A}=\boldsymbol{O}$ which was considered in $[3$, Theorem 5.1].

Theorem 3. Let $G_{2}^{1} \in \mathcal{C}^{0, v}(\Gamma)$, with $v>\frac{d}{2 n}$. Then the jump the problem (2) has a solution explicitly given by

$$
\begin{gathered}
\boldsymbol{\Xi}_{\mathbf{2}}^{\mathbf{1}}=\widetilde{\mathbf{G}}_{2}^{1}-\frac{(-1)^{\frac{n(n+1)}{2}}}{(2 i)^{n}} \mathcal{T}_{\mathbf{\Omega}}^{\mathcal{A}} \mathcal{D}_{\left(\underline{\underline{Z}}, \underline{Z}^{\dagger}\right)}^{\mathcal{A}} \widetilde{G}_{2}^{1}, \text { in } \Omega_{+} \\
\boldsymbol{\Xi}_{\mathbf{2}}^{\mathbf{1}}=-\frac{(-1)^{\frac{n(n+1)}{2}}}{(2 i)^{n}} \mathcal{T}_{\mathbf{\Omega}}^{\mathcal{A}} \mathcal{D}_{\left(\underline{\underline{Z}}, \underline{Z}^{\dagger}\right)}^{\mathcal{A}} \widetilde{G}_{2}^{1}, \text { in } \Omega_{-}
\end{gathered}
$$

Proof.

On account of the assumption on $v$, it follows that $\mathcal{D}_{\left(\underline{Z}, \underline{Z}^{\dagger}\right)}^{\mathcal{A}} \widetilde{G}_{2}^{1}$ belongs to $\mathbf{L}_{p}(\Omega)$, for $p>2 n$. Then, the Hölder continuity of $\Xi_{2}^{1}$ in $\Omega_{ \pm} \cup \Gamma$ directly follows from Theorem 1, (i) and (iii). Next, the matrix inversion formula (ii) in Theorem 1 yields

$$
\mathcal{D}_{\left(\underline{Z}, \underline{Z}^{\dagger}\right)}^{\mathcal{A}} \boldsymbol{\Xi}_{2}^{\mathbf{1}}=O \text { in } \mathbb{R}^{2 n} \backslash \Gamma
$$

showing the $\mathbf{H}^{\mathcal{A}}$-monogenicity of $\boldsymbol{\Xi}_{2}^{\mathbf{1}}$ in $\Omega_{ \pm}$. Finally, the validity of the jump condition (2) is a matter of direct verification. 


\subsection{Treatment of the problem (3)-(4)}

The aim of this subsection is to apply the previously introduced techniques in order to treat the boundary value problem (3)-(4).

Theorem 4. Let $G_{2}^{1} \in \mathcal{C}^{0, v}(\Gamma)$, with $v>\frac{d}{2 n}$. If there exists $\mathbf{\Xi}_{\mathbf{2}}^{\mathbf{1}} \in \mathcal{C}^{0, v}(\bar{\Omega})$ such that

$$
\left\{\begin{array}{c}
\mathcal{D}_{\left(\underline{Z}, \underline{Z}^{\dagger}\right)}^{\mathcal{A}} \boldsymbol{\Xi}_{\mathbf{2}}^{\mathbf{1}}=O, \text { in } \Omega \\
\boldsymbol{\Xi}_{\mathbf{2}}^{\mathbf{1}}=G_{2}^{1}, \text { on } \Gamma
\end{array}\right.
$$

then

$$
\left.\left[\mathcal{T}_{\mathbf{\Omega}}^{\mathcal{A}} \mathcal{D}_{\left(\underline{Z} \underline{Z}^{\dagger}\right)}^{\mathcal{A}} \widetilde{G}_{2}^{1}\right]\right|_{\Gamma}=\mathbf{O} .
$$

Conversely, if (8) is satisfied, then there exists a function $\boldsymbol{\Xi}_{\mathbf{2}}^{\mathbf{1}} \in \mathcal{C}^{0, \mu}(\bar{\Omega})(\mu<v)$ satisfying (7).

Proof.

Assume (7) to hold and fix $\Psi_{2}^{\mathbf{1}}=\widetilde{\mathbf{G}}_{2}^{1}-\boldsymbol{\Xi}_{2}^{\mathbf{1}}$. Obviously, $\mathbf{\Psi}_{\mathbf{2}}^{\mathbf{1}} \in \mathcal{C}^{0, v}(\bar{\Omega})$ and $\left.\mathbf{\Psi}_{2}^{\mathbf{1}}\right|_{\Gamma}=\mathbf{O}$. But

$$
\mathcal{T}_{\Omega}^{\mathcal{A}} \mathcal{D}_{\left(\underline{Z}, \underline{Z}^{\dagger}\right)}^{\mathcal{A}} \widetilde{G}_{2}^{1}=\mathcal{T}_{\Omega}^{\mathcal{A}} \mathcal{D}_{\left(\underline{Z}, \underline{Z}^{\dagger}\right)}^{\mathcal{A}} \Psi_{2}^{1}
$$

since $\mathcal{T}_{\Omega}^{\mathcal{A}} \mathcal{D}_{\left(\underline{\underline{Z}}, \underline{\underline{Z}}^{\dagger}\right)}^{\mathcal{A}} \widetilde{\Xi}_{2}^{1}=\boldsymbol{O}$.

We are thus reduced to proving that $\mathcal{T}_{\Omega}^{\mathcal{A}} \mathcal{D}_{\left(\underline{Z}, \underline{Z}^{+}\right)}^{\mathcal{A}} \widetilde{\mathbf{\Psi}}_{2}^{1}=\boldsymbol{O}$ on $\Gamma$. To this end, define

$$
\Omega_{k}=\left\{\underline{X} \in Q \mid Q \in \mathcal{W}^{j}, \text { for some } j \leq k\right\}
$$

It simplifies the argument, and causes no loss of generality, to assume that $\Omega_{k}$ is connected. Observe that the boundary of $\Omega_{k}$, denoted $\Gamma_{k}$, consists of certain faces of some cubes $Q \in \mathcal{W}^{k}$. We then have

$$
\begin{aligned}
\int_{\Omega} \mathcal{E}_{A}(\underline{Z}-\underline{V}) \mathcal{D}_{\left(\underline{Z} \underline{Z}^{\dagger}\right)}^{\mathcal{A}} \widetilde{\Psi}_{2}^{1}(\underline{X}) d W\left(\underline{Z}, \underline{Z}^{\dagger}\right) \\
\quad=\lim _{k \rightarrow \infty} \int_{\Omega_{k}} \mathcal{E}_{A}(\underline{Z}-\underline{V}) \mathcal{D}_{\left(\underline{Z}, \underline{Z}^{\dagger}\right)}^{\mathcal{A}} \widetilde{\Psi}_{2}^{1}(\underline{X}) d W\left(\underline{Z}, \underline{Z}^{\dagger}\right)
\end{aligned}
$$

Now, take $\underline{Y} \in \Omega$ and choose $k_{0}$ sufficiently large, such that $\underline{Y} \in \Omega_{k_{0}}$ and $\operatorname{dist}\left(\underline{Y}, \Gamma_{k}\right) \geq \frac{\left|Q_{0}\right|}{2 \sqrt{2 n}}$ for $k>k_{0}, Q_{0}$ being a cube of $\mathcal{W}^{k_{0}}$. The Hermitean BorelPompeiu formula (see [1, Proposition 1]), applied to $\Omega_{k}$, then yields

$$
\begin{gathered}
(-1)^{\frac{n(n+1)}{2}}(2 i)^{n} \widetilde{\mathbf{\Psi}}_{2}^{1}(\underline{Y})+\int_{\Omega_{k}} \mathcal{E}_{A}(\underline{Z}-\underline{V}) \mathcal{D}_{\left(\underline{Z} \underline{Z}^{+}\right)}^{\mathcal{A}} \widetilde{\mathbf{\Psi}}_{2}^{1}(\underline{X}) d W\left(\underline{Z}, \underline{Z}^{\dagger}\right) \\
=\int_{\Gamma_{k}} \mathcal{E}_{A}(\underline{Z}-\underline{V}) \mathbf{N}_{\left(\underline{Z}, \underline{Z}^{+}\right)}^{k} \widetilde{\mathbf{\Psi}}_{2}^{1}(\underline{X}) d \mathcal{H}^{2 n-1}
\end{gathered}
$$

where $\mathbf{N}_{\left(\underline{Z}, \underline{Z}^{\dagger}\right)}^{k}$ is the circulant matrix corresponding to the unit normal vector on $\Gamma_{k}$. Next, consider $\underline{X} \in \Gamma_{k}$, let $Q \in \mathcal{W}^{k}$ be a cube containing $\underline{X}$, and take $\underline{P} \in \Gamma$ such that $|\underline{X}-\underline{P}|=\operatorname{dist}(\underline{X}, \Gamma)$. Since $\left.\widetilde{\Psi}_{2}^{1}\right|_{\Gamma}=0$, we have

$$
\left\|\widetilde{\mathbf{\Psi}}_{2}^{1}(\underline{X})\right\|=\left\|\widetilde{\mathbf{\Psi}}_{2}^{1}(\underline{X})-\widetilde{\mathbf{\Psi}}_{2}^{1}(\underline{P})\right\| \leq c|\underline{X}-\underline{P}|^{v} \leq c|Q|^{v}
$$


If $\Sigma$ denotes a face of $\Gamma_{k}$ and $Q \in \mathcal{W}^{k}$ is the $k$-cube containing that face $\Sigma$, then we have, for $k>k_{0}$, that

$$
\begin{aligned}
\left\|\int_{\Sigma} \mathcal{E}_{A}(\underline{Z}-\underline{V}) \mathbf{N}_{\left(\underline{Z}, \underline{Z}^{\dagger}\right)}^{k} \widetilde{\mathbf{\Psi}}_{2}^{1}(\underline{X}) d \mathcal{H}^{2 n-1}\right\| & \leq \frac{c}{\left|Q_{0}\right|^{2 n-1}} \int_{\Sigma}\left\|\widetilde{\mathbf{\Psi}}_{2}^{1}(\underline{X})\right\| d \mathcal{H}^{2 n-1} \\
& \leq \frac{c}{\left|Q_{0}\right|^{2 n-1}}|Q|^{v-1+2 n}
\end{aligned}
$$

Since each face of $\Gamma_{k}$ belongs to some $Q \in \mathcal{W}^{k}$, we have, for $k>k_{0}$,

$$
\begin{aligned}
\left\|\int_{\Gamma_{k}} \mathcal{E}_{A}(\underline{Z}-\underline{V}) \mathbf{N}_{\left(\underline{Z}, \underline{Z}^{+}\right)}^{k} \widetilde{\mathbf{\Psi}}_{2}^{1}(\underline{X}) d \mathcal{H}^{2 n-1}\right\| & \leq \frac{c}{\left|Q_{0}\right|^{2 n-1}} \sum_{Q \in \mathcal{W}^{k}}|Q|^{v-1+2 n} \leq \\
& \leq \frac{c}{\left|Q_{0}\right|^{2 n-1}} \sum_{Q \in \mathcal{W}^{k}}|Q|^{d} .
\end{aligned}
$$

The finiteness of the $d$-sum $\sum_{Q \in \mathcal{W}}|Q|^{d}$ of the Whitney decomposition $\mathcal{W}$ of $\Omega$, see Lemma 2, implies

$$
\lim _{k \rightarrow \infty} \int_{\Gamma_{k}} \mathcal{E}_{A}(\underline{Z}-\underline{V}) \mathbf{N}_{\left(\underline{Z}, \underline{Z}^{+}\right)}^{k} \widetilde{\mathbf{\Psi}}_{2}^{1}(\underline{X}) d \mathcal{H}^{2 n-1}=\mathbf{O} .
$$

Combining (9) with (10) yields $\left.\mathcal{T}_{\Omega}^{\mathcal{A}} \mathcal{D}_{\left(\underline{Z}, \underline{Z}^{+}\right)}^{\mathcal{A}} \widetilde{\mathbf{\Psi}}_{2}^{1}\right|_{\Gamma}=\boldsymbol{O}$.

On the contrary, if (8) holds the assertion follows directly by taking $\boldsymbol{\Xi}_{2}^{\mathbf{1}}=\widetilde{\mathbf{G}}_{2}^{1}-\mathcal{T}_{\mathbf{\Omega}}^{\mathcal{A}} \mathcal{D}_{\left(\underline{Z}, \underline{Z}^{\dagger}\right)}^{\mathcal{A}} \widetilde{G}_{2}^{1}$.

The following direct consequence of Theorem 4 give representation formulas for the solutions of the homogeneous perturbed Hermitean Dirac equation under Dirichlet conditions.

Corollary 1. Let $G_{2}^{1} \in \mathcal{C}^{0, v}(\bar{\Omega})$, with $v>\frac{d}{2 n}$ be $\mathbf{H}^{\mathcal{A}}$-monogenic in $\Omega$ with trace $\boldsymbol{g}_{2}^{1}$ on $\Gamma$, then

$$
\boldsymbol{G}_{2}^{1}(\underline{X})=\tilde{\boldsymbol{g}}_{2}^{1}(\underline{X})-\mathcal{T}_{\Omega}^{\mathcal{A}} \mathcal{D}_{\left(\underline{Z} \underline{Z}^{\dagger}\right)}^{\mathcal{A}} \tilde{\boldsymbol{g}}_{2}^{1}(\underline{X}), \underline{X} \in \Omega .
$$

We finish with a result concerning the solvability of the problem (3)-(4).

Theorem 5. Let $\boldsymbol{G}_{2}^{1} \in \mathcal{C}^{0, v}(\Gamma)$ and $\boldsymbol{F}_{2}^{1} \in \mathbf{L}^{p}(\Omega)$ such that $v$ and $\frac{p-2 n}{p}$ are both greater that $\frac{d}{2 n}$. If there exists a function $\boldsymbol{\Xi}_{\mathbf{2}}^{\mathbf{1}} \in \mathcal{C}^{0, v}(\bar{\Omega})$ solution of the problem (3)-(4), then

$$
\left.\left[\mathcal{T}_{\mathbf{\Omega}}^{\mathcal{A}} \mathcal{D}_{\left(\underline{Z}, \underline{Z}^{\dagger}\right)}^{\mathcal{A}} \widetilde{G}_{2}^{1}\right]\right|_{\Gamma}=\left.\left[\mathcal{T}_{\mathbf{\Omega}}^{\mathcal{A}} \boldsymbol{F}_{2}^{1}\right]\right|_{\Gamma}
$$

Conversely, if (12) is satisfied, then there exists a function $\boldsymbol{\Xi}_{\mathbf{2}}^{\mathbf{1}} \in \mathcal{C}^{0, \mu}(\bar{\Omega})(\mu<v)$ solution of the problem (3)-(4).

Proof.

We first take $\Theta_{2}^{1}=\Xi_{2}^{1}-\mathcal{T}_{\Omega}^{\mathcal{A}} \boldsymbol{F}_{2}^{1}$. Assuming $\Xi_{2}^{\mathbf{1}}$ be a solution of (3)-(4) we see that $\boldsymbol{\Theta}_{2}^{1}$ is a solution of the homogeneous problem (7) with $G_{2}^{1}$ replaced by $\boldsymbol{G}_{2}^{1}-\left.\left[\mathcal{T}_{\boldsymbol{\Omega}}^{\mathcal{A}} \boldsymbol{F}_{2}^{1}\right]\right|_{\Gamma} \in \mathcal{C}^{0, \beta}(\Gamma)$, where

$$
\beta:=\min \left\{v, \frac{p-2 n}{p}\right\}
$$


It is easy to check that $\Phi_{2}^{1}:=\widetilde{\boldsymbol{G}}_{2}^{1}-\mathcal{T}_{\Omega}^{\mathcal{A}} \boldsymbol{F}_{2}^{1}-\boldsymbol{\Theta}_{2}^{1} \in \mathcal{C}^{0, \beta}(\bar{\Omega})$ such that $\left.\Phi_{2}^{1}\right|_{\Gamma}=\boldsymbol{O}$.

By assumption, $\beta>\frac{d}{2 n}$, then as in the proof of Theorem 4 we have that $\left.\mathcal{T}_{\Omega}^{\mathcal{A}} \mathcal{D}_{\left(\underline{Z}, \underline{Z}^{\dagger}\right)}^{\mathcal{A}} \widetilde{\Phi}_{2}^{1}\right]\left.\right|_{\Gamma}=\boldsymbol{O}$ and hence that

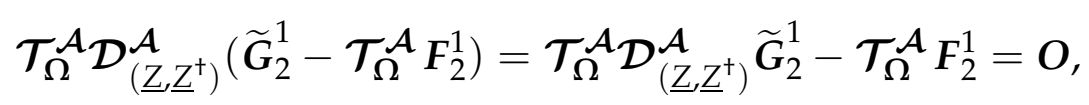

which proves (12).

The second assertion follows directly by taking

$$
\boldsymbol{\Xi}_{2}^{\mathbf{1}}=\widetilde{\mathbf{G}}_{2}^{1}-\mathcal{T}_{\Omega}^{\mathcal{A}} \mathcal{D}_{\left(\underline{\underline{Z}}, \underline{\underline{Z}}^{\dagger}\right)}^{\mathcal{A}} \widetilde{G}_{2}^{1}+\mathcal{T}_{\Omega}^{\mathcal{A}} F_{2}^{1}
$$

Corollary 2. Let $\boldsymbol{G}_{2}^{1} \in \mathcal{C}^{0, v}(\Gamma)$ and $\boldsymbol{F}_{2}^{1} \in \mathbf{L}^{p}(\Omega)$ such that $v$ and $\frac{p-2 n}{p}$ are both greater that $\frac{d}{2 n}$. If $\boldsymbol{\Xi}_{\mathbf{2}}^{\mathbf{1}} \in \mathcal{C}^{0, v}(\bar{\Omega})$ has trace $G_{2}^{1}$ on $\Gamma$ and satisfies $\mathcal{D}_{\left(\underline{Z}, \underline{Z}^{+}\right)}^{\mathcal{A}} \boldsymbol{\Xi}_{\mathbf{2}}^{\mathbf{1}}=\boldsymbol{F}_{2}^{1}$ on $\Omega$, then

$$
\boldsymbol{\Xi}_{\mathbf{2}}^{\mathbf{1}}(\underline{X})=\widetilde{\mathbf{G}}_{2}^{1}(\underline{X})-\mathcal{T}_{\mathbf{\Omega}}^{\mathcal{A}} \mathcal{D}_{\left(\underline{Z}, \underline{Z}^{\dagger}\right)}^{\mathcal{A}} \widetilde{\boldsymbol{G}}_{2}^{1}(\underline{X})+\mathcal{T}_{\mathbf{\Omega}}^{\mathcal{A}} \boldsymbol{F}_{2}^{1}(\underline{X}), \underline{X} \in \Omega .
$$

\section{Concluding Remark}

We have been working under the assumption that the surface of the considered domain is fractal, which makes the study of boundary value problems rather complex.

The results obtained in the present work continue the line of research offered in [1] and provide us with a mathematical basis for the use of the Teodoresco transform associated to the perturbed Hermitean matrix Dirac operator for the treatment of boundary value problems in the case of fractal boundaries.

\section{Acknowledgments}

The research carried out in this paper was completed during Ricardo Abreu stay as Visiting Professor at Universidade Federal de Alagoas, Maceió, supported by "Programa Profesor Visitante do Exterior -PVE/CAPES", Projeto 10699139. The financial support and kind hospitality are gratefully acknowledged.

The authors would like to express their thanks to the referee for his/her valuable comments and suggestions, which greatly helped to improve the quality of the paper.

\section{References}

[1] R. Abreu Blaya; J. Bory Reyes; F. Brackx; H. De Schepper; F. Sommen. Boundary value problems associated to a Hermitean Helmholtz equation. J. Math. Anal. Appl., 389 (2), 1268-1279, 2012.

[2] R. Abreu Blaya; J. Bory Reyes; F. Brackx; H. De Schepper; F. Sommen. A Hermitean Cauchy formula on a domain with fractal boundary. J. Math. Anal. Appl., 369, 273-282, 2010. 
[3] R. Abreu Blaya; J. Bory Reyes; F. Brackx; H. De Schepper. Hermitean Téodorescu transform decomposition of continuous matrix functions on fractal hypersurfaces. Bound. Value Probl., vol. 2010, Article ID 791358, 15 pages, 2010.

[4] R. Abreu Blaya; J. Bory Reyes; F. Brackx; H. De Schepper; F. Sommen. A Hilbert transform for matrix functions on fractal domains. Compl. Anal. Oper. Theory, 6 (2), 359-372, 2012.

[5] S. Bernstein. Fundamental solutions for Dirac-type operators. Lawrynowicz, J. (ed.), Generalizations of complex analysis and their applications in physics. Warszawa: Polish Academy of Sciences, Inst. of Mathematics, Banach Cent. Publ., 37, 159-172, 1996.

[6] F. Brackx; R. Delanghe; F. Sommen. Clifford analysis, Research Notes in Mathematics 76, Pitman (Advanced Publishing Program), Boston, 1982.

[7] F. Brackx; J. Bureš; H. De Schepper; D. Eelbode; F. Sommen; V. Souček. Fundaments of Hermitean Clifford analysis. I. Complex structure. Complex Anal. Oper. Theory 1, no. 3, 341-365, 2007.

[8] F. Brackx; J. Bureš; H. De Schepper; D. Eelbode; F. Sommen; V. Souček. Fundaments of Hermitean Clifford analysis. II. Splitting of h-monogenic equations. Complex Var. Elliptic Equ. 52, no. 10-11, 1063-1079, 2007.

[9] F. Brackx; H. De Schepper; F. Sommen. The Hermitean Clifford analysis toolbox. Adv. Appl. Clifford Algebras, 18, no. 3-4, 451-487, 2008.

[10] F. Brackx; B. De Knock; H. De Schepper. A matrix Hilbert transform in Hermitean Clifford analysis. J. Math. Anal. Appl. 344, 1068-1078, 2008.

[11] F. Brackx; B. De Knock; H. De Schepper; F. Sommen. On Cauchy and Martinelli-Bochner integral formulae in Hermitean Clifford analysis. Bull Braz Math Soc. 40 (3), 395-416, 2009.

[12] K. J. Falconer. The geometry of fractal sets, Cambridge Tracts in Mathematics 85, Cambridge Univ. Press, Cambridge, 1986.

[13] H. Federer. Geometric measure theory, Springer Verlag, New York Inc., New York, 1969.

[14] K. Gürlebeck; W. Sprössig. Quaternionic and Clifford Calculus for Physicists and Engineers, Wiley and Sons Publ., 1997.

[15] K. Gürlebeck; M. Shapiro; W. Sprössig. On a Teodorescu transform for a class of metaharmonic functions. J. Nat. Geom. 21, no. 1-2, 17-38, 2002.

[16] Vu Thi Ngoc Ha. Higher order Teodorescu operators in quaternionic analysis related to the Helmholtz operator. Math. Nachr. 280, No. 11, 1268-1281, 2007.

[17] J. Harrison, A. Norton, The Gauss Green theorem for fractal boundaries, Duke Math. J. 67 (3), 575-588, 1992. 
[18] V. V. Kravchenko. Applied Quaternionic Analysis, Heldemann Verlag, Berlin, 2003.

[19] M.L. Lapidus, H. Maier. Hypothèse de Riemann, cordes fractales vibrantes et conjecture de Weyl-Berry modifiée, C.R. Acad. Sci. Paris Série I Math., 313 (1), 19-24, 1991.

[20] A. MacIntosh; M. Mitrea. Clifford Algebras and Maxwell's Equations in Lipschitz Domains. Math. Methods Appl. Sci., 22, 1599-1620, 1999.

[21] M. Mitrea. Boundary Value Problems and Hardy Spaces Associated to the Helmholtz Equation in Lipschitz Domains. J. Math. Anal. Appl., 202, 819-842, 1996.

[22] R. Rocha-Chávez; M. Shapiro; F. Sommen. Integral theorems for functions and differential forms in $\mathbb{C}^{m}$. Research Notes in Mathematics, 428. Chapman \& Hall/CRC, Boca Raton, FL, 2002.

[23] E.M. Stein, Singular Integrals and Differentiability Properties of Functions, Princeton Math. Ser. 30, Princeton Univ. Press, Princeton, N.J., 1970.

[24] X. Zhenyuan. A function theory for the operator $D-\lambda$. Complex Variables, Theory Appl., 16 1, 27-42, 1991.

Facultad de Informática y Matemática,

Universidad de Holguín,

Holguín 80100, Cuba.

Email: rabreu@facinf.uho.edu.cu

Departamento de Matemática, Universidad de Oriente,

Santiago de Cuba 90500, Cuba

Email: jbory@rect.uo.edu.cu 